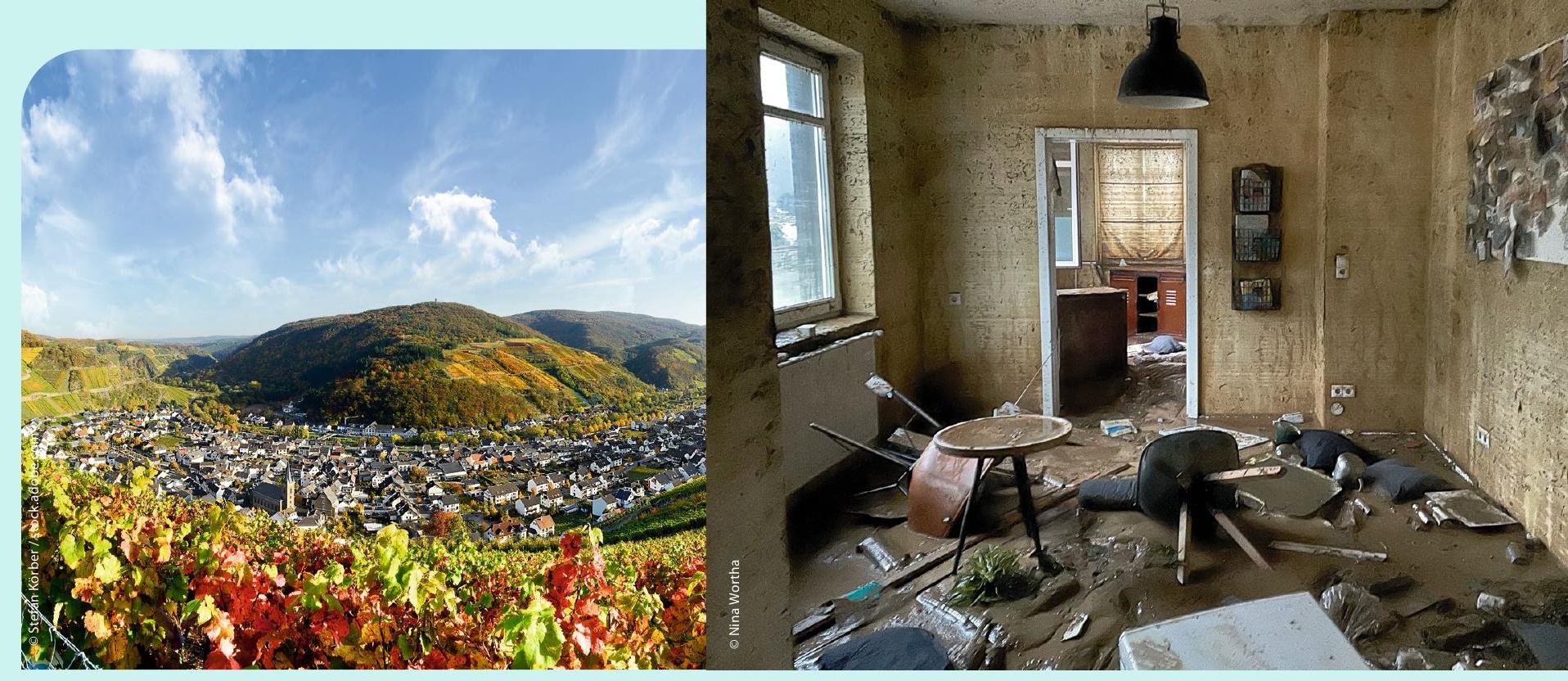

\title{
Hausbesuch nach der Flut
}

\author{
Hebammenbetreuung im Ahrtal Der Pegel der Ahr stieg Mitte Juli rasant, es \\ ging nicht um Stunden, sondern um wenige Minuten. Die Flutkatastrophe hat \\ viele Menschen das Leben gekostet und zahlreiche Existenzen ruiniert - auch \\ von Hebammen. Doch aufgeben gibt es nicht - denn Schwangere und Wöch- \\ nerinnen brauchen jetzt mehr denn je eine fürsorgliche Betreuung.
}

$\mathbf{V}$ on der Geschwindigkeit, mit der das Wasser kam, und der Wucht der Wassermassen war man besonders im Ahrtal total überrascht. Auch Hebamme Marion Sermann wird von den Wassermassen überrumpelt - daheim und in den neuen Praxisräumen, die sie im Mai 2020 erst mit ihrer Kollegin Nina Wortha in Dernau bezogen hat. Sermann selbst hatte am Hochwassertag einen Bürotag zuhause und hätte nur abends einen Kurs gegeben. „Den Kurs habe ich tatsächlich schon im Laufe des Tages abgesagt, weil meine Familie ein Weingut im Ahrtal hat und mein Lebensgefährte nach Dienstschluss zum Hochwasserschutz dorthin fahren wollte und meine Tochter dann keine Betreuung gehabt hätte“, erklärt die freiberufliche Hebamme. Ihre Kundinnen zeigten Verständnis, wünschten für die Familie alles Gute und gaben der Hoffnung Ausdruck, dass es nicht so schlimm werde. Zu dem Zeitpunkt ahnten viele nicht, welche Katastrophe sie erwartete. Das zeigt auch ein Telefonat Sermanns mit ihrer Kollegin Wortha im Laufe des Nachmittags. Diese hatte sich mit ihrem Mann zusammen um die Praxis gekümmert: „Wir haben alles mit Sandsäcken gesichert. Mach dir keine Sorgen, das sind bestimmt noch vier bis fünf Meter von der Ahr bis hoch zu den Praxisräumen. Das wird nichts. Kümmere du dich jetzt um dein Haus.“

\section{Der Wasserpegel stieg sehr schnell}

Denn dort war schon früh Land unter - und das, obwohl Sermann nicht direkt an der Ahr, sondern in einem Teil von Altenahr wohnt, wo es noch einen kleinen Bach gibt. Sie fing an, sich allmählich Sorgen zu machen, als es nicht aufhörte zu regnen und „der Abfluss anfing, bedenklich zu gluckern“. Um 18 Uhr war das Haus komplett umflutet und ihr Keller bis unter die Decke voll mit Wasser. „Ich hätte am Folgetag um 10 Uhr einen Rückbildungskurs gehabt, den habe ich dann direkt abgesagt“, erinnert sie sich. Zu diesem Zeitpunkt wusste sie noch gar nicht, was woanders los war. „Bereits am Abend waren wir vom Internet abgeschnitten“, schildert sie diese ersten Stunden. Sie bekam nicht mit, dass Leute festsaßen oder auf ihren Dächern sitzend um Hilfe schrieen.

Nachdem Sermann und ihr Lebensgefährte ihren Keller aufgegeben und sich am nächsten Morgen um 6 Uhr im Ort einen ersten Eindruck der Gesamtlage verschafft hatten, versuchte sie, mit ihrer Kollegin Wortha, die in der Grafschaft wohnt und dort Netz, Strom, Wasser und alles noch hatte, Kontakt aufzunehmen. „Nina hat dann auch meine werdenden Mütter kontaktiert und ihnen geholfen. Ich hatte drei Frauen direkt hier im Ort, so dass ich die erste Woche nur mit meiner Tasche zu Fuß unterwegs war. Meine Kol- 
Oben (v.I.n.r.): Pure Idylle im Ahrtal. Als die Flut kam zerstörte sie alles - auch die Hebammenpraxis "Zwergenmanufaktur". Rechts: Vorher: Es steckte viel Arbeit und Herzblut in der Hebammenpraxis in Dernau. Die Schwangeren fühlten sich hier sehr wohl.

legin hat alle anderen Frauen sowie alles rund um die Praxis übernommen. Denn dort stand das Wasser ebenfalls bis unter der Decke“, schildert die junge Hebamme. „Wir haben alles verloren, die Einrichtung, die Buchhaltungsdokumente, das Material. Gott sei Dank haben wir mit Laptops gearbeitet, die nicht in der Praxis waren. Zudem dokumentieren wir seit 2019 alles online. So sind wenigstens unsere Akten nicht draufgegangen“, berichtet Sermann. Und trotzdem ist sie sich bewusst, dass sie Glück hatte und alle ihre Freunde und die Familie wohlauf sind und sie weiterhin privat ein Dach über dem Kopf hat. Es ist für sie bis heute dramatisch, das Ausmaß der Zerstörung zu sehen, wenn sie mit dem Auto unterwegs ist.

\section{Alltag nach der Katastrophe}

Nach zehn Tagen hat Sermann begonnen, wieder die ersten Hausbesuche zu fahren - mit Umwegen und längeren Fahrzeiten, aber es ging. „Wir machen seitdem alles, was wir bisher in der Praxis gemacht haben, nur eben im Hausbesuch oder vereinzelt auch online“, schildert sie den neuen Arbeitsalltag. Sie gibt aber auch zu, dass Straßensperrungen, Ampeln oder nicht mehr vorhandene Brücken sie in ihrer Berufsausübung momentan nicht allzu sehr behindern. Sie legt sich ihre Termine so, dass sie nicht zur Hauptverkehrszeit weite Strecken zurücklegen muss. „Jetzt muss ich auch erst einmal schauen, denn beispielsweise die Hauptverkehrsstraße ins Ahrtal verläuft durch einen Tunnel, hinter dem sich nun ein riesiger Krater auftut. Die Orte, die danach kommen, sind für mich natürlich nicht mehr direkt anfahrbar", beschreibt die Hebamme, die demnächst aber auch dort wieder Hausbesuche machen will.

Bei Anja Huser, angestellter Hebamme am Krankenhaus „Maria Hilf“ in Bad Neuenahr, stellt sich die Situation etwas anders dar: „Ich musste gestern zu drei Frauen, habe um 10 Uhr das Haus verlassen und war um 17 Uhr wieder zurück. Es sind nicht nur die Wege, die mehr Zeit in Anspruch nehmen, auch die Frauen benö-

tigen momentan die Zeit", weiß sie zu berichten. Das Erlebte sei eben auch psychisch für viele sehr belastend. „Die ersten 14 Tage gingen Tag und Nacht die Martinshörner der Helferfahrzeuge, ununterbrochen flogen die Hubschrauber - erst für die Luftrettung, dann zur Leichenbergung. Doch am stärksten hat sich mir ins Gedächtnis gebrannt, dass Panzer an unserem Garten vorbei gerollt sind“, erinnert sich Huser. Langsam kann man aber immerhin das Ahrtal wieder passieren, das war lange Zeit gar nicht möglich. „Wenn ich zur Arbeit wollte, wohl gemerkt eigentlich nur 8,5 km entfernt, bin ich über die Autobahn $42 \mathrm{~km}$ unterwegs gewesen“, schildert Huser. Für sie war und ist Hilfe keine Frage: Ihre Kinder sind fast erwachsen, sie hat ein Auto und „dann fahr ich halt“, ist ihre Antwort.

Wie alle in den betroffenen Gebieten hat auch die freiberufliche Hebamme Marion Sermann gerade in der Anfangszeit in erster Linie funktioniert. Sie hat versucht, ihre Frauen, die aufgrund des Hochwassers eben nicht mehr vor Ort waren, über Facebook bei anderen Hebammen unterzubringen, um diese weiterhin gut versorgt zu wissen. Zudem hatte sie für alle Belange ein offenes Ohr und hat in Not geratenen Familien zu Spenden verholfen. „Oft hat es schon an den kleinsten Dingen gefehlt“, weiß sie zu berichten. Neben der beruflichen Verantwortung war für Sermann auch die private Hochwasser-Situation herausfordernd: „In der ersten Nacht haben mein Lebensgefährte und ich auf dem Sofa geschlafen und sind alle halbe Stunde patrouilliert, ob das Wasser im Erdgeschoss im Rahmen bleibt. Neben der Arbeit als Hebamme habe ich dann noch bei meiner Familie auf dem Weingut geholfen." Jetzt kommt, nach vielen Wochen, wieder eine Routine auf: Sermann fährt regulär am Wochenende ihre Hausbesuche und beginnt ihren nächsten Geburtsvorbereitungskurs am Abend. Dennoch merkt man ihr die Sorge und Anspannung der vergangenen Wochen deutlich an. Erst langsam begreift sie, dass die Praxis nicht mehr da ist: „Ich kann nicht mehr mal eben in die Praxisräume fahren, um schnell 


\section{† UNTERSTÜTZUNG FÜR BETROFFENE HEBAMMEN}

\begin{abstract}
Die Bilder der Flutkatastrophe haben viele Menschen in Deutschland geschockt. Neben zahlreicher privat organisierter Spendenaufrufe hat auch der Landesverband der Hebammen Nordrhein-Westfalen zusammen mit dem Deutschen Hebammenverband und der Hebammengemeinschaftshilfe Unterstützungsangebote organisiert. Diese reichen von Spendenaufrufen, über die Koordinierung praktischer Hilfen aus dem Bundesgebiet - z.B. über die Vermittlungsplattform ammely.de bis hin zu ersten finanziellen Hochwasser-Hilfen für betroffene Hebammen. Die Flutkatastrophe hat jedoch nicht nur materiellen Schaden und physische Spuren hinterlassen. Daher bietet beispielsweise die BGW auch verschiedene psychologische Formen der Betreuung auch für Hebammen - in dieser Extremsituation an.
\end{abstract}

\section{hebammen-nrw.de}

etwas zu holen, was ich beispielsweise für eine Vorsorgeuntersuchung benötige."

\section{Unbeschreiblicher Zusammenhalt}

Die beiden Jungunternehmerinnen sind daher sehr froh über das Angebot einer befreundeten Praxis in Meckenheim, in der sie sich einklinken dürfen. „Das ist ein tolles Angebot gerade auch für Frauen, die zu weit weg wohnen und die wir derzeit eben nicht anfahren können. Viele unserer Frauen wollen aber auch die Hebammen nicht wechseln, weil die Babys gerade geboren sind“, schildert sie den Freundschaftsdienst. Auch der Kontakt untereinander und mit dem Hebammenverband hat sehr geholfen, „um den Druck rauszunehmen, weil wir wussten, die Frauen sind erst einmal versorgt“". Denn am Anfang war die Angst groß, dass ein Großteil der gynäkologischen Praxen nicht mehr arbeiten kann, erinnern sich Sermann und Huser. In der Region hat die gynäkologische Abteilung, in der Huser arbeitet, binnen kürzester Zeit ein gut funktionierendes System aufgebaut, so dass werdende Mütter und Notfälle auch ohne gynäkologische Praxis weiterhin gut versorgt waren. „Alle schwangeren Frauen in der Region konnten dann zu uns ins Krankenhaus zur Kontrolle kommen“, sagt Huser. Denn: „Es gab im Stadtgebiet Bad Neuenahr anfänglich keine gynäkologische ambulante Versorgung." Dabei ist die Finanzierung noch ungeklärt, weil das Krankenhaus ja keinen Kassensitz hat und damit eigentlich auch nicht abrechnen kann.

„Gleich zu Beginn hat und die Hebammenpraxis Jork kontaktiert und gefragt, was wir brauchen, um arbeiten zu können. Die haben gesammelt und sind zehn Tage später angereist und haben uns eine Tasche mit genau diesen Sachen gebracht, so dass wir schon früh wieder die Schwangerenvorsorge durchführen konnten“, schildert Sermann sichtlich ergriffen. Auch ein Anruf des Geburtshauses Bielefeld ist fest in ihrer Erinnerung verankert: „Die kamen mit drei Kolleginnen ins Ahrtal, um zu helfen, und standen in Dernau vor unserer Praxis, haben das Ausmaß der Verwüstung gesehen und einfach bei uns angerufen: „Wir haben alles dabei für Vorsorge, Nachsorge, Geburt. Wenn ihr irgendwas braucht, dann sagt Bescheid“, waren ihre Worte, die uns in der Situation wieder Lichtblicke verschafft haben." Daneben gab es noch zahlreiche weitere Hilfsangebote, die die beiden freiberuflichen Hebammen bekommen und die sie tief beeindruckt haben.

Sermann kann retrospektiv sogar Corona etwas Positives abgewinnen, denn eine „Online-Leistungserbringung ist ja normalerweise gar nicht möglich“. Sie hofft darauf, dass die Krankenkassen den freiberuflich tätigen Hebammen Corona-bedingt auch in diesem Winter noch das Onlinekurs-System weiter zugestehen werden. Dann wären die Sorgen wegen der nun fehlenden Praxisräume für die Kurse auch erst einmal aus dem Kopf.

\section{Notversorgung im Kreißsaal}

Anja Huser hat auch ordentlich mit angepackt: Obwohl das Krankenhaus zwischenzeitlich evakuiert war, hat sie sich dafür eingesetzt, dass der Kreißsaal für Notfälle 24 Stunden besetzt blieb. „Für eine werdende Mutter war es tatsächlich zu spät, sie noch zu verlegen oder woanders hinzuschicken. Ich wollte sie eigentlich in ein anderes Krankenhaus begleiten. Doch als ich ankam, war das Kind schon da. Ich habe eigentlich wie gesagt nur 8,5 km bis zum Arbeitsplatz, aber ich hing in einer Kolonne fest“, erklärt sie. Dreißig angemeldete Geburten für den Juli wurden jedoch abgesagt.

Auch viele ehemalige Wöchnerinnen von Huser hat es hart getroffen. Eine von ihnen ist mit ihrer Familie eine Woche vor dem Hochwasser aus dem höher gelegenen Teil von Rech in ihr neues Haus eingezogen - mit ihren vier Kindern. „Ich musste mich sortieren, als ich auf Rech hinuntergeschaut habe, denn ich habe die Kirche, das Pfarrhaus und das Schulgebäude gesehen, die man normalerweise gar nicht von dem Punkt aus sehen kann, weil andere Häuser davor stehen. Und dann dachte ich nur: Was fehlt denn hier alles?", erinnert sich die Hebamme mit Schrecken.

Es gibt aber auch noch Dinge, die fehlen, die man aber nicht sieht, beispielsweise eine warme Heizung: Die Fernwärme ist in manchen Regionen erst wieder für Januar bzw. Februar avisiert. „Da kann man eigentlich nicht wohnen bleiben, den Winter ohne Heizung. Und erst recht nicht hochschwanger oder mit einem Neugeborenen." Jedoch sehen das viele Betroffene anders, schildert Huser die Situation vor Ort. Sie besorgen sich alternative Heizmöglichkeiten und bleiben in ihren Häusern.

Seit Mitte August dürfen wieder Babys in Krankenhaus „Maria Hilf“ das Licht der Welt erblicken. Huser selbst hatte in ihrem ersten regulären Dienst - in der Nacht vom 12. auf den 13. August eine Mutter, die entbunden hat, die seit dem 4. August gewartet hatte. "Sie wollte unbedingt bei uns entbinden und hat sich so gefreut, dass es wieder möglich war", berichtet Huser gerührt. \I

Alexandra Heeser 
Hier steht eine Anzeige.

黛 Springer 